\title{
The Impact of Stellar Rotation on the Nitrogen Abundance Gradient from OB Stars in the Galactic Disk
}

\author{
Simone Daflon and Katia Cunha \\ Observatório Nacional/MCT, R. Gal. José Cristino 77, São Cristovão, \\ 20921-400 Rio de Janeiro/RJ, Brazil
}

\begin{abstract}
Massive young stars can be used to trace the current chemical composition of the Galactic disk and to define its metallicity gradient. However, evolutionary models of massive rotating stars predict changes of the surface abundances due to rotationally induced mixing. The abundance analysis of elements sensitive to mixing, such as CNO, can be used to test these rotating stellar models. In this study we identify those stars that show one particular signature of mixing, N-enrichment, in our sample of $70 \mathrm{OB}$ stars for which we have conducted an abundance analysis. This sample is used to define radial metallicity gradients in the Milky Way and we investigate the variation of the calculated nitrogen gradient after the exclusion of these, probably mixing-induced, N-rich stars.
\end{abstract}

\section{Introduction}

Stellar rotation increases the main sequence lifetime and changes surface abundances of massive stars. Heger \& Langer (2000) computed a grid of models of stars with $\mathrm{M}=8-20 \mathrm{M}_{\odot}$ including rotation and predicted changes in surface abundances of CNO caused by rotationally induced mixing. From the observational point of view Daflon et al. (2001) found N-enrichment of a factor $\sim 2$ in the two most evolved stars from a sample of 17 main-sequence OB star members of the Cep OB2 association. The evidence that rotation can induce mixing and produce high nitrogen surface abundances, even on the main sequence, raises the need to check if the nitrogen abundances in our sample of OB stars are all primordial, or whether some have been altered by mixing; such stars should be excluded from any sample used to study nitrogen abundance gradients.

\section{Identifying the N-Rich Stars}

We derived non-LTE abundances of several elements in $70 \mathrm{OB}$ main-sequence stars, including stars with low- and high- projected rotational velocities (Daflon 2000). Our sample contains stars with $v \sin i$ between 7 to $190 \mathrm{~km} \mathrm{~s}^{-1}$ and is baised towards sharp-lined stars, because such stars are easier to analyze for abundances: 43 stars of the sample have $v \sin i<60 \mathrm{~km} \mathrm{~s}^{-1}$. This stellar sample lies within $4.4<R_{g}<12.9 \mathrm{kpc}$ from the Galactic center and the chemical abundances are derived from the fitting of broadened non-LTE synthetic line profiles to high resolution observed spectra. The non-LTE calculations were done 

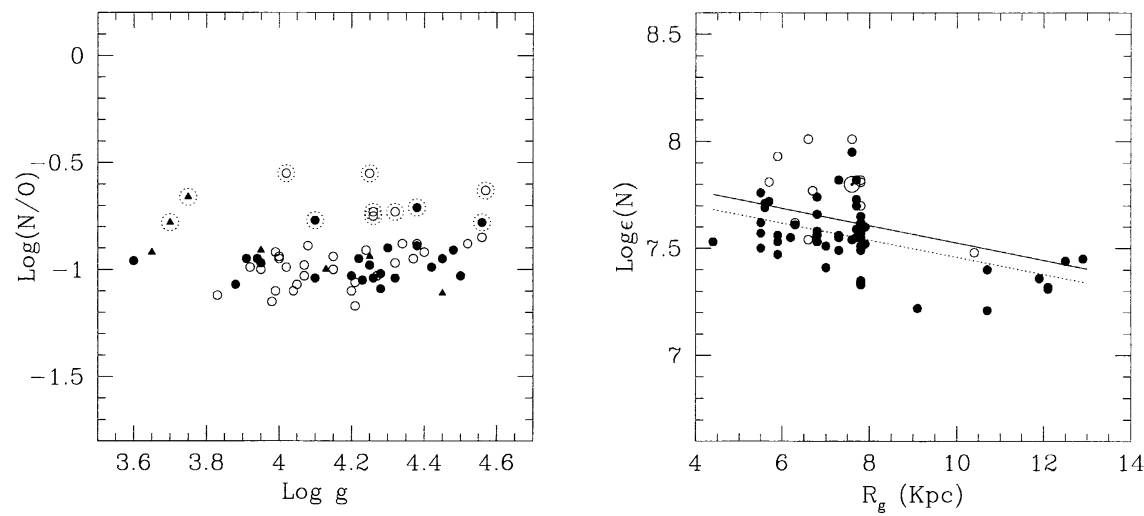

Figure 1. Left panel: $\mathrm{N} / \mathrm{O}$ ratios as a function of $\log g$. Different $v \sin i$ are represented by different symbols: $1<v \sin i<49$ (open circle), $50<v \sin i<$ 99 (filled circle), and $v \sin i>100$ (filled triangle) $\mathrm{km} \mathrm{s}^{-1}$. Stars marked with a dotted circle are considered N-enriched. Rigth panel: Nitrogen abundances as a function of Galactocentric distance. Filled symbols are for stars with normal N/O and open symbols are for N-rich stars. The solid line shows the gradient derived for the 70 stars in the sample and the dotted line represents the gradient obtained when the N-rich stars are discarded.

with programs DETAIL, which solves simultaneously the equations of statistical equilibrium and transfer, and SURFACE, which computes the line profiles.

In order to investigate the impact of mixing on the radial distribution of $\mathrm{N}$ abundances in our sample we inspect their $\mathrm{N} / \mathrm{O}$ ratios as a function of the surface gravity in Figure 1 (left panel). We identify as N-enriched those stars with $\mathrm{N} / \mathrm{O}<-0.8$ (marked with a dotted circle in the Figure). Inspection of their $\log$ g's shows that there is evidence of $\mathrm{N}$-enrichment on the main sequence and that this enrichment is found among stars with both low- and high- $v \sin i$. In the right panel of Figure 1 we show the radial gradient obtained for nitrogen considering the 70 stars in our sample: a slope of $-0.042 \pm 0.012 \mathrm{dex} / \mathrm{Kpc}$. With the exclusion of those stars identified as mixed in the left panel of Figure 1 we obtain a gradient of $-0.039 \pm 0.011 \mathrm{dex} / \mathrm{Kpc}$.

\section{References}

Daflon, S., Cunha, K., Butler, K. \& Smith, V. V. 2001, ApJ 563, 325

Daflon, S. 2002, Ph.D. Thesis, Observatório Nacional/MCT

Heger, A., \& Langer, N. 2000, ApJ, 544, 1016 